\title{
Intrinsic Viscosity and Friction Coefficient of Polymer Molecules in Solution: Porous Sphere Model
}

\author{
P. F. MIJNLIEFF and F. W. WIEGEL, Department of Applied Physics, \\ Twente University of Technology, Enschede, The Netherlands
}

\section{Synopsis}

The intrinsic viscosity $[\eta]$ and the translational friction coefficient $f$ of polymer molecules in solution are calculated on the basis of the porous sphere model. The only information needed to predict $[\eta]$ and $f$ is the polymer molecular weight, the radius of gyration in the solvent, and the permeability as a function of position in the "porous sphere." For systems for which this information is available there is satisfactory agreement between predicted and directly measured values of $[\eta]$ and $f$. No adjustment of parameters is required. The influence of solvent quality is more complex than is suggested by the experimentally verified Flory-Fox relation for $[\eta]$; the simple form of this relation stems from the fact that two quite large effects of solvent quality approximately compensate each other. The complete flow pattern of the solvent around and through the polymer coil can be calculated. Contrary to what is usually believed the solvent flow in the polymer coil is not "effectively blocked", even at the center. 'The connection between the present treatment and the microscopic theory of Kirkwood and Riseman is investigated.

\section{INTRODUCTION}

Well known quantities, used to characterize polymer molecules in solution are $[\eta]$, the intrinsic viscosity, and $f$, the translational friction coefficient of the polymer molecules at infinite dilution. The determination of $[\eta]$ and $f$ from viscosity and sedimentation measurements, respectively, is relatively simple. However, in the interpretation of the measured values in terms of polymer molecular weight, radius of gyration, and, in particular, solvent flow through and around the polymer coils, some questions have remained unanswered.

Almost invariably the molecular theory of Kirkwood and Riseman ${ }^{1,2 a}$ is taken as a starting point in this interpretation. In the Kirkwood-Riseman theory the degree of hydrodynamic interaction (influence of nearby as well as more remote parts of the polymer chain on the solvent flow at a certain point) is expressed by the value of the so-called draining parameter $h$, which may run from zero (no hydrodynamic interaction) to infinity (dominant hydrodynamic interaction). The parameter $h$ is* a combination of quantities that occur in the molecular model. It is almost impossible to derive their value, and therefore that of $h$, from the chemical structure of the particular polymer and solvent considered.

For this reason $h$ is often treated as an adjustable parameter and the value assigned to it is the one that makes theoretical and experimental values of $[\eta]$ and $f$ agree.

It then appears that all flexible polymers, whatever the quality of the solvent, show a behavior that corresponds to "dominant hydrodynamic interaction" $(h$ $\rightarrow \infty$ ). To be more explicit, measured $[\eta]$ and $f$ values can be converted into so-called effective hydrodynamic radii by means of the relations

$$
R_{\text {eff,visc }}=\left(\frac{3}{10 \pi N_{\mathrm{Av}}}\right)^{1 / 3}(M[\eta])^{1 / 3}
$$

* For a definition of $h$ see ref. 2(a), eq. (31.23). 
and

$$
R_{\text {eff,trans }}=\left(1 / 6 \pi \eta_{0}\right) f
$$

where $N_{\mathrm{Av}}$ is Avogadro's number, $M$ is the polymer molecular weight, and $\eta_{0}$ is the viscosity of the solvent.

Now, for the limiting case of "dominant hydrodynamic interaction," the Kirkwood-Riseman theory leads to effective radii that are related to $\rho_{g}$, the radius of gyration, as ${ }^{3 a}$

$$
R_{\text {eff, visc }}=0.87 \rho_{g}
$$

and $^{3 b}$

$$
R_{\text {eff,trans }}=0.66 \rho_{g}
$$

It is found that the values of the effective radii as calculated from measured [ $\eta]$ and $f$ values by means of eqs. (1) and (2) are very close to, if not above, the values for the limit of "dominant hydrodynamic interaction" given in eqs. (3) and (4). This empirical fact is well represented by the famous Flory-Fox equation: ${ }^{4 a}$

$$
[\eta]=\Phi \rho_{g}^{3} M^{-1}
$$

in which the constant $\Phi$ has the same solvent-independent value for all flexible polymers.

The simplicity of these results (any deviations from eq. (5) only appear in a closer analysis) cannot easily be explained from an estimate of the quantities occurring in the Kirkwood-Riseman theory. As it was pointed out ${ }^{4 \mathrm{~b}}$ by Kurata and Stockmayer, "unreasonably large friction coefficients per segment" have to be used in order for the limit of dominant hydrodynamic interaction, in the sense explained above, to be reached. Regardless of the failure of the explanations, they are forced ${ }^{4 c}$ to accept the experimental evidence for this being the situation; a particularly puzzling aspect is the almost complete lack of influence of the solvent on the degree of hydrodynamic interaction. After ten year ${ }^{2 b}$ the analysis of experimental results still appears to lead to essentially the same conclusion.

In the present paper the "degree of draining" and related questions are rediscussed. This time, however, on the basis of calculations starting from a semimacroscopic model, the "porous sphere" model, proposed by Debye and Bueche $^{5,6}$ and by Brinkman. ${ }^{7,8}$

For reasons explained below, this model was not used or further evaluated for a long time. Some years ago, however, it was shown ${ }^{9}$ that it did produce correct values for the friction coefficient $f$ without an $a$ posteriori adjustment of parameters.

Using the same model we now have calculated $f$ in a somewhat different way and added a calculation for $[\eta]$. Predicted $f$ and $[\eta]$ values appear to be in satisfactory agreement with experimental data. This means, among other things, that we do indeed predict that the effective hydrodynamic radii are about equal to the radii of gyration, in theta solvents as well as in good solvents. It appears, however, that this fact is not the consequence of a general scaling law, but the result of there being two effects. Each effect, if present alone, would seriously violate the validity of eq. (5). Their joint influence upon the hydrodynamic radii, however, offsets any singular effect and, just by chance, they approximately 
compensate. We think this is the explanation for the rather unexpected general validity of eq. (5).

The following section deals with the model used, with the basic equations, and with the basic data used in the calculations. The calculations themselves, with the exception of what is essential for the present paper, are reported elsewhere. ${ }^{10}$ The results of the calculation of $f$ and $[\eta]$ and a comparison between theory and experiment are given in the Results section. Also given are some results on the flow (draining) of the solvent through the polymer coil, as it occurs in a viscosity measurement and in a sedimentation experiment. This is followed by conclusions and discussion.

\section{THE MODEL: BASIC EQUATIONS AND ESSENTIALS OF THE CALCULATION}

\section{The Model}

Both $[\eta]$ and $f$, owing to the way they are measured, reflect the macroscopic response of a polymer solution to a stimulus (applied rate of shear and applied field strength). It is the response, therefore, of a collection of macromolecules with conformations distributed according to some law. Considering linear processes only, we take for this distribution law the one that corresponds to thermodynamic equilibrium. This is also the distribution "developed" by each individual polymer molecule in the course of time. And, roughly speaking, the conformations through which a polymer molecule passes during a time interval of the order of magnitude of its longest characteristic time already form a set representative of the distribution containing all possible conformations.

Superimposed to the thermal motion, always present, the molecule may carry out a motion (rotation or translation) imposed by the macroscopic boundary conditions (simple-shear flow or parallel flow). As in the microscopic Kirkwood-Riseman theory the latter motion will be described as taking place according to the laws of macroscopic hydrodynamics. In addition to this, however, we shall apply macroscopic hydrodynamics, not to every polymer molecule conformation separately, but to a fictitious object (a modeled polymer molecule) which is already some kind of "averaged" molecule; the construction of this "average" molecule is specified later.

To justify this approach it may be noted that, by making the external stimulus weaker and weaker, the imposed motion can always be made negligible as compared with thermal motion. More specifically, if the time needed for a molecule to make one revolution owing to the applied rate of shear, or to travel, due to the applied field, over a distance equal to its own (average) diameter is long in comparison with the longest characteristic time of the molecule, the laws of hydrodynamics, being applicable only to molecular motions averaged over a certain time interval, apply to the "average" molecule rather than to a molecule in any instantaneous conformation.

Whereas the above conditions can always be satisfied by making the external stimulus sufficiently weak, it can easily be shown that for polymer molecules with a longest characteristic time (relaxation time, for example) as large as $10^{-2} \mathrm{sec}$ they are already satisfied in the usual viscosity and ultracentrifuge experiments. 
Additional and less qualitative support for applying hydrodynamics to the average polymer comes from the microscopic theory. Before discussing this point, however, we specify the choice of the "averaged polymer molecule." This is the object constructed in the following way: the polymer molecules with all the conformations that together form the equilibrium distribution of conformations are imagined to be superimposed on each other in such a way that their centers of gravity coincide. The resulting, spherically symmetric, continuous distribution of mass is then normalized so that

$$
\int_{0}^{\infty} c(r) 4 \pi r^{2} d r=m_{1}
$$

where $c$ is the local concentration (mass per unit volume) of polymer material in the resulting cloud, $r$ is the distance from the center of gravity, and $m_{1}$ is the molecular mass of the polymer. This cloud is the "averaged polymer molecule" cited above. Next, to any concentration $c$ a certain value of the permeability $k$ is assigned. A certain permeability distribution $k(r)$ therefore corresponds to the averaged polymer molecule. The notion of permeability was introduced by Darcy in connection with the flow of liquids through a porous material, and the representation of a polymer molecule by a permeability distribution $k(r)$ is what is called the "porous sphere model." Debye ${ }^{5}$ and Brinkman 7,8 independently suggested the use of this model for calculating frictional properties of polymer molecules in solution.

Permeability can be defined in various equivalent ways. We will use it as it occurs in Darcy's law

$$
\mathbf{V}-\mathbf{U}=-(k / \eta) \operatorname{grad} P
$$

for the flow of a Newtonian liquid with viscosity $\eta$ through a plug of rigid porous material under the influence of grad $P$, the gradient of the hydrostatic pressure $P$ in the liquid. Here $\mathbf{U}$ and $\mathbf{V}$ are, respectively, the velocity of the plug and the "average" velocity of the liquid (volume of liquid flowing per second through a plane of unit area perpendicular to $\operatorname{grad} P$ ).

\section{Basic Equations}

For calculating $[\eta]$ and $f$ it will appear to be necessary to know $\mathbf{V}_{0}$, the (averaged) local solvent velocity, as a function of place. Again Debye ${ }^{5,6}$ as well as Brinkman ${ }^{7,8}$ proposed to calculate $\mathbf{V}_{0}$ from the differential equation (DebyeBrinkman equation)

$$
-\operatorname{grad} P+\eta_{0} \operatorname{div} \operatorname{grad} \mathbf{V}_{0}-\left[\eta_{0} / k(r)\right]\left(\mathbf{V}_{0}-\mathbf{U}\right)=0
$$

in combination with the incompressibility expression

$$
\operatorname{div} \mathbf{V}_{0}=0
$$

Equation (8) is a combination of the linearized Navier-Stokes equation for the stationary situation, characterized by

$$
\frac{\partial \mathbf{V}_{0}}{\partial t}=0
$$

and the Darcy equation. 
The physical background of eq. (8) is discussed elsewhere. ${ }^{11}$ The equation was used by Debye and Bueche ${ }^{6}$ and by Brinkman ${ }^{7}$ for calculating the frictional coefficients $[\eta]$ and $f$ of a porous sphere with a uniform permeability $k$. For a long time, however, it remained unused for predicting $[\eta]$ and $f$ for real polymer molecules. The main reason for this was that no way seemed to exist to calculate from first principles or to derive from independent measurements the $k(r)$ data needed to solve eq. (8).

The molecular theory of Kirkwood and Riseman ${ }^{1}$ appears to lead to a similar, stagnant situation. Recently the molecular approach was picked up again by Felderhof and Deutch. ${ }^{12-15}$ They were able to show, ${ }^{12}$ by carrying out certain averagings, that the mean solvent velocity $\mathbf{V}_{0}$ through and around the polymer, for the stationary state characterized by eq. (10), was described by the differential equation

$$
-\operatorname{grad} P+\eta_{0} \operatorname{div} \operatorname{grad} \mathbf{V}_{0}-\rho_{p} \zeta\left(\mathbf{V}_{0}-\mathbf{U}\right)=0
$$

again in combination with eq. (9). The quantities $\rho_{p}$ and $\zeta$ are, respectively, the local number-density of the (effective) segments in the polymer molecule and the effective translational friction coefficients of one of these segments. The analogy between eqs. (11) and (8) is the molecular support, referred to above, for the porous sphere model; the quantity $\rho_{p} \zeta$, occurring in eq. (11), corresponds to the quantity $\eta_{0} / k(r)$, occurring in eq. (8).

To date no prediction of $\rho_{p}$ and $\zeta$ (or of their product) on a molecular basis seems to be possible; in this respect the situation is the same as it is for the draining parameter $h$. Some years ago, however, it was shown ${ }^{16}$ that the $k(r)$ data needed to solve eq. (8), could be derived straightforwardly from separate measurements. Primarily these measurements provide us with $k(c)$, the per-

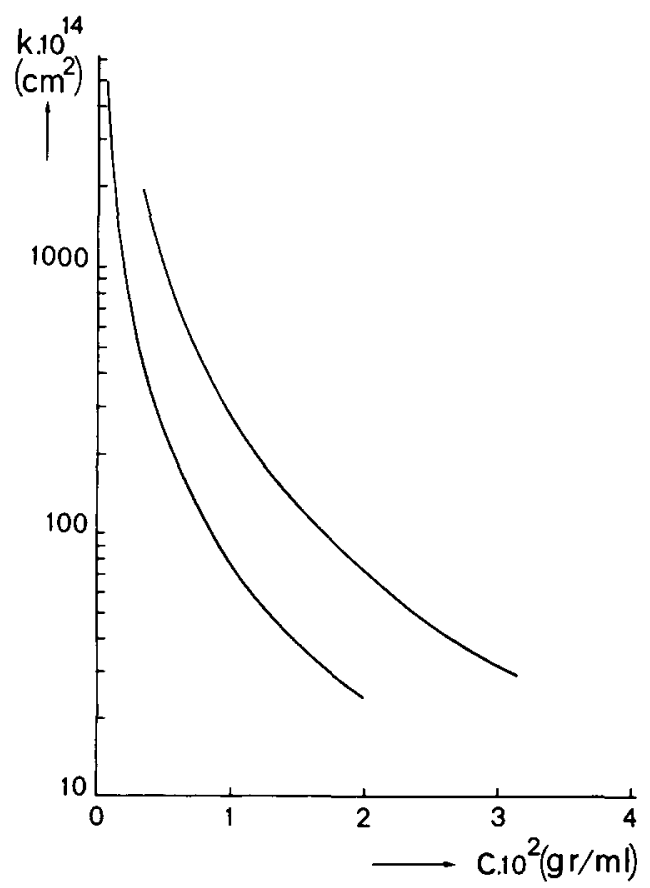

Fig. 1. Permeability as a function of concentration in system A (upper curve) and in system B (lower curve). Systems A and B are defined in Results section. 
meability as a function of concentration of polymer. Some typical results are shown in Figure 1.

The function $k(c)$, in particular its magnitude, appears to depend strongly on the quality of the solvent for the particular polymer, permeability being lower for the better solvent. By combining $k(c)$ data, as contained in Figure 1, with a function $c(r)$, as meant in eq. (6), the $k(r)$ data are obtained and eq. (8) can be solved.

Some years ago this was done ${ }^{9}$ for the boundary conditions prevailing in sedimentation experiments, and the solutions were used for calculating $f$. There was a good agreement between $f$ thus predicted and values found directly from sedimentation coefficients at zero polymer concentration.

For $c(r)$, the function

$$
c(r)=\frac{M_{1}}{N_{\mathrm{Av}}}\left(\frac{2}{3} \pi \rho_{\mathrm{g}}^{2}\right)^{-3 / 2} \exp \left(-\frac{3 r^{2}}{2 \rho_{g}^{2}}\right)
$$

was adopted. The same expression will be used in the present paper. Certainly for polymer molecules obeying random flight statistics eq. (12) is a fair approximation. $^{2 c}$

Combination of the $k(c)$ curves of Figure 1 with the $c(r)$ curves representing eq. (12), leads to $k(r)$ curves that appear to be reasonably well described by the two-parameter expression

$$
k(r)=K \exp \left(Q r^{2}\right)
$$

From eqs. (12) and (13) it follows that

$$
K=k(r=0)=k(c(r=0))=k\left(c=\frac{M}{N_{\mathrm{Av}}}\left(\frac{2}{3} \pi \rho_{g}{ }^{2}\right)^{-3 / 2}\right)
$$

Furthermore, the permeability $k$ (shown in Fig. 1) appears to be almost inversely proportional to $c$, certainly in the low concentration region. For this reason we say:

$$
Q=3 / 2 \rho_{g}{ }^{2}
$$

Equation (8) will be solved for a permeability $k(r)$ described by eq. (13); from eqs. (14) and (15) it is seen that, given a certain molecular mass, the parameters which must be known are $k(c(r=0))$ and $\rho_{g}$.

\section{Essentials of the Calculation}

Only the most essential points of the calculation of $[\eta]$ and $f$ will be mentioned. A more complete account is given elsewhere. ${ }^{10}$ To arrive at $[\eta]$, a polymer solution is imagined between two infinite parallel plates separated by a fixed distance $2 L$ and moving at constant relative velocity.* The particle (polymer molecule) centers are chosen to be the origins of Cartesian coordinate systems, all oriented in such a way that the $y$ direction is perpendicular to the plates and the $x$ direction is parallel to the direction of the motion of the plates. All particle centers will, on the average, move parallel to the plates, with a velocity somewhere between the velocities of the two plates. With respect to any particle, therefore, one plate, say the plate with the positive $z$ coordinate, moves in the positive and

\footnotetext{
* We call the separation distance $2 L$, and not $L$, in conformity with notation elsewhere. ${ }^{10}$
} 
the other plate in the negative $x$ direction. We denote the positive velocity difference between the plates by $\Delta V_{p, x}$, and the forces to be exerted per unit area on the plates to maintain their steady-state motion by $+k_{x}$ and $-k_{x}$. The viscosity of the solution $\eta$ is then defined as

$$
\eta \equiv k_{x} / G
$$

in which $G$, defined as

$$
G \equiv \Delta V_{p, x} / 2 L
$$

is called the average rate of shear.

When the liquid between the plates is pure solvent, the liquid assumes a uniform simple-shear flow, having a rate of shear $d V_{0, x} / d y$ that equals $G$.

We now consider the case of a solution sufficiently dilute for the plates to have material contact with pure solvent only. Then the force $k_{x}$ may also be looked upon as the force needed to maintain a rate of shear equal to* $\left(d V_{0, x} / d y\right)_{p}$ in the solvent layer adjoining the plates; from this point of view we may write, according to continuum mechanics:

$$
k_{x}=\eta_{0}\left(\frac{d V_{0, x}}{d y}\right)_{p}
$$

Another consequence of considering dilute solutions is that the solvent flow far away $(r \rightarrow \infty)$ from any individual particle is still a simple-shear flow, albeit with a rate of shear $G_{e}$ that differs slightly from the average rate of shear $G$.

The plates being far from all particles, the rate of shear $\left(d V_{0, x} / d y\right)_{p}$ in eq. (18) is equal to this $G_{e}$. . Using this result we find from eqs. (16) and (18) that

$$
\eta G=\eta_{0} G_{e}
$$

Because $[\eta]$ is defined as

$$
[\eta] \equiv\left(\frac{\eta-\eta_{0}}{\eta_{0} c_{1}}\right)_{c_{1} \rightarrow 0}
$$

where $c_{1}$ is the polymer concentration (mass per unit volume), the quantity to be calculated is $G_{e} / G$.

From the above it will be clear that eq. (8), together with eq. (9), has to be solved with boundary conditions

$$
\begin{gathered}
V_{0, x, r \rightarrow \infty}=G_{e} y \\
V_{0, y, r \rightarrow \infty}=0 \\
V_{0, z, r \rightarrow \infty}=0
\end{gathered}
$$

if the Cartesian coordinate system has its origin in the particle considered. If the particles, like the polymer molecules in the porous sphere model, possess spherical symmetry, it can be shown that their motion becomes a stationary rotation about the $z$ axis with an angular velocity $\omega$ given by

$$
\omega=-1 / 2 G_{e}
$$

This means that the components of $\mathbf{U}$, the local velocity of the porous material, to be introduced into eq. (8), are

\footnotetext{
* Here we restrict ourselves to a solvent rate of shear not varying along a plate, for this will be the case actually studied (see eq. (21)).
} 


$$
\begin{gathered}
U_{x}=-\omega y=1 / 2 G_{e} y \\
U_{y}=+\omega x=-1 / 2 G_{e} x \\
U_{z}=0
\end{gathered}
$$

The manner in which eqs. (8) and (9), subject to conditions (21) and (22), are solved is treated elsewhere. ${ }^{10}$ At this point we only mention that $V_{0, x}$ becomes

$$
V_{0, x}=G_{e} y-G_{e} A\left(x^{2} y / r^{5}\right)+O\left(r^{-4}\right)
$$

in which $A$ is a constant determined by $K$ and $Q$ :

$$
A \equiv \alpha Q^{-3 / 2} \Phi(\alpha)
$$

with

$$
\alpha \equiv K^{-1} Q^{-1}
$$

and where $\Phi(\alpha)$, a function to be calculated numerically, contains $\alpha$ as a parameter. This function is shown in Figure 2.

From eq. (23) we calculated $[\eta]$ by a procedure due to Burgers. ${ }^{17}$ In this procedure a system containing $n_{p}$ foreign particles per unit volume is considered. The aim of the procedure is to calculate $\Delta V_{0, x}$, the difference between solventvelocity components in the $x$ direction (far from the particles to the only remaining one) at points separated by a distance $2 L$ in the $y$ direction. The result, obtained using eq. (23) for $V_{0, x}$, reads: ${ }^{10}$

$$
\Delta V_{0, x}=2 L\left(1-4 / 3 \pi n_{p} A\right) G_{e}
$$

If there is no slip between the plates and the adjoining liquid, $\Delta V_{0, x}$ is equal to $\Delta V_{p, x}$, occurring in eq. (17). Thus, combining eqs. (17), (19), and (26), and introducing the relation

$$
n_{p}=\left(N_{\mathrm{Av}} / M\right) c_{1}
$$

we obtain

$$
\frac{\eta}{\eta_{0}}=\frac{1}{1-4 / 3 \pi\left(N_{\mathrm{Av}} / M\right) c_{1} A}
$$

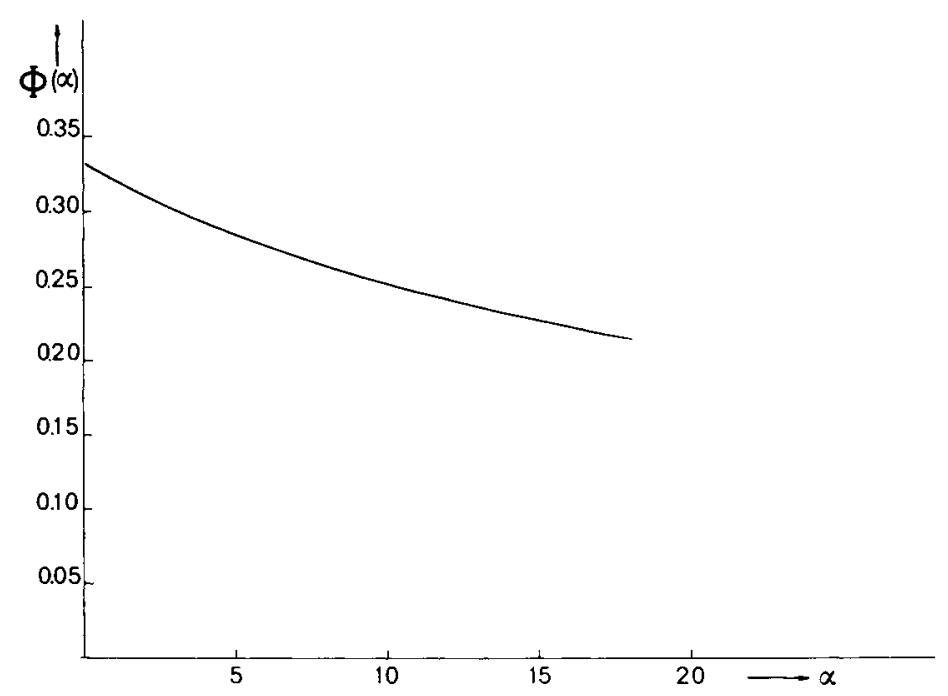

Fig. 2. Plot of $\Phi$, occurring in eq. (24), vs. $\alpha$, defined in eq. (25). 
Introducing this result into eq. (20) and applying eq. (24) we find:

$$
[\eta]=4 / 3 \pi\left(N_{\mathrm{Av}} / M\right) \alpha Q^{-3 / 2} \Phi(\alpha)
$$

This result will be used the Results section for calculating $[\eta]$.

In addition to the "global" property $[\eta]$, the (complete) solution of eqs. (8) and (9) provides us with all the information on the solvent flow around and through the polymer coil. Some complete velocity profiles are given elsewhere. ${ }^{10}$ Here we consider only one interesting quantity, viz.,

$$
\left(\frac{d}{d y} V_{0, x}(x=0, z=0)\right)_{\text {at } y=0}
$$

which is the velocity gradient of the solvent at the center of the coil. From the calculations it appears that

$$
\left(\frac{d}{d y} V_{0, x}(x=0, z=0)\right)_{\text {at } y=0}=\{1 / 2+1 / 2 f(r=0)\} G_{e}
$$

The quantity $f(r=0)$ is obtained in the process of solving eq. (8) numerically. It contains $\alpha$ as a parameter. It runs from zero, when $\alpha=\infty$ (permeability zero), to unity when $\alpha=0$ (permeability infinite), and is a measure for the degree of draining for the following reason. When $f(r=0)$ is zero, then, according to eq. (28), the shear rate of the solvent at the coil center is $1 / 2 G_{e}$. In view of eq. (22), for $U_{x}$ this means that the solvent does not move relative to the coil (no draining). On the other hand, when $f(r=0)$ is unity, then, again according to eq. (28), the solvent shear rate at the center equals $G_{e}$. This means that the velocity profile of the solvent is completely unaffected by the coil (complete draining). Reality, as it will be shown below, lies about halfway between these extremes.

About the calculation of $f$ we will be very brief. Equations (8) and (9) are solved by considering the polymer coil to be fixed $(\mathbf{U}=\mathbf{0})$, whereas the solvent distant from it moves (at constant speed) in the positive $z$ direction. That is:

$$
\begin{gathered}
V_{0, x, r \rightarrow \infty}=0 \\
V_{0, y, r \rightarrow \infty}=0 \\
V_{0, z, r \rightarrow \infty}=V_{0}
\end{gathered}
$$

Solution of eqs. (8) and (9) for these conditions leads to a velocity profile with the $z$ component given by ${ }^{10}$

$$
V_{0, z}=V_{0}-V_{0} \frac{C}{2 r}-V_{0} \frac{C z^{2}}{2 r^{3}}+O\left(r^{-3}\right)
$$

in which $C$ is related to the friction coefficient: ${ }^{10}$

$$
f=4 \pi \eta_{0} C
$$

Like the quantity $A$ in the case of viscosity, the value of $C$ is connected with the complete solution of eq. (8). The relevant calculation is again given elsewhere. ${ }^{10}$ The result can be written (cf., eq. (24)) in the form

$$
C=Q^{-1 / 2} \alpha \Psi(\alpha)
$$

so that

$$
f=4 \pi \eta_{0} Q^{-1 / 2} \alpha \Psi(\alpha)
$$




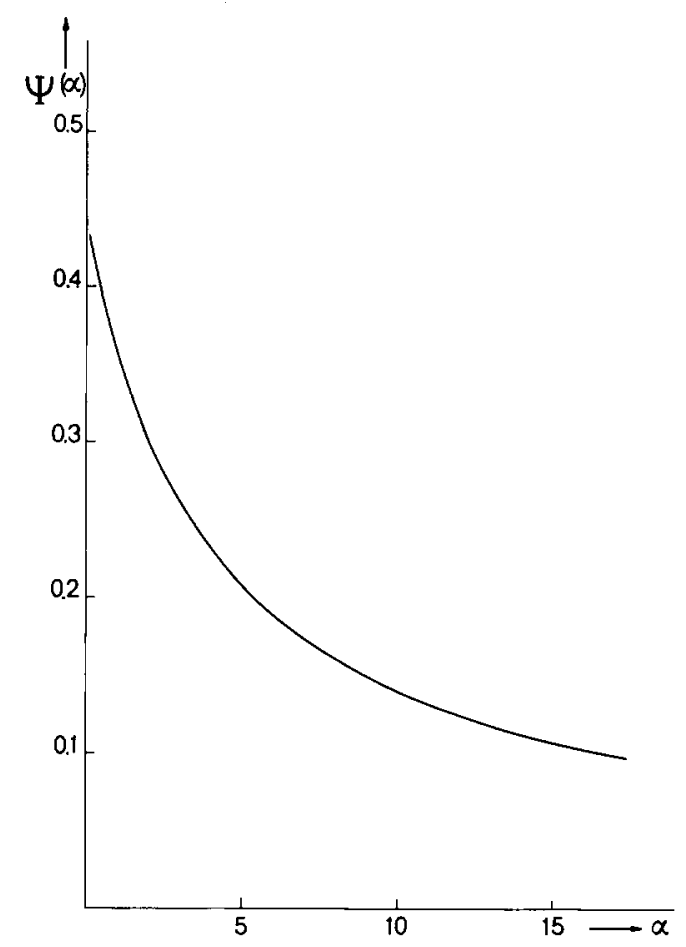

Fig. 3. Plot of $\Psi$, occurring in eq. (30), vs. $\alpha$, defined in eq. (25).

The function $\Psi(\alpha)$, calculated numerically, is shown in Figure 3. In the Results section eq. (30) will be used to predict $f$.

Also contained in the complete solution is the pattern of the solvent flow through and around the coil. Some complete patterns are given elsewhere. ${ }^{10}$ Here we consider only the quantity

$$
V_{0, z}(x=0, y=0, z=0) / V_{0} \equiv V_{0, \text { rel }}
$$

where is a measure of draining at the center of the coil. Specific results are given later.

\section{RESULTS OF CALCULATIONS AND COMPARISON WITH EXPERIMENT}

In this section values of $[\eta]$ and $f$ for some real polymer-solvent systems are calculated and compared with those obtained by direct measurements of these quantities in the same system. Furthermore, some results on the effective hydrodynamic radii and the degree of drainage are given.

The basic expressions $[\eta]$ and $f$ are eqs. (27) and (30). In view of the meaning of $Q, \alpha$, and $\mathrm{K}$ given in eqs. (15), (25), and (14), these expressions allow us to predict $[\eta]$ and $f$ for polymer molecules, where the permeability curves $k(c)$ and the radii of gyration $\rho_{g}$ as a function of molecular weight, are known for the solvents to be considered. Polymer-solvent systems for which this is the case and for which, in addition, experimental values of $[\eta]$ and $f$ are available are: (system A) poly $(\alpha$-methylstyrene $)$ in cyclohexane at $35.5^{\circ} \mathrm{C}$-a system at the theta temperature - and (system B) poly $\left(\alpha\right.$-methylstyrene) in toluene at $25^{\circ} \mathrm{C}$-an 


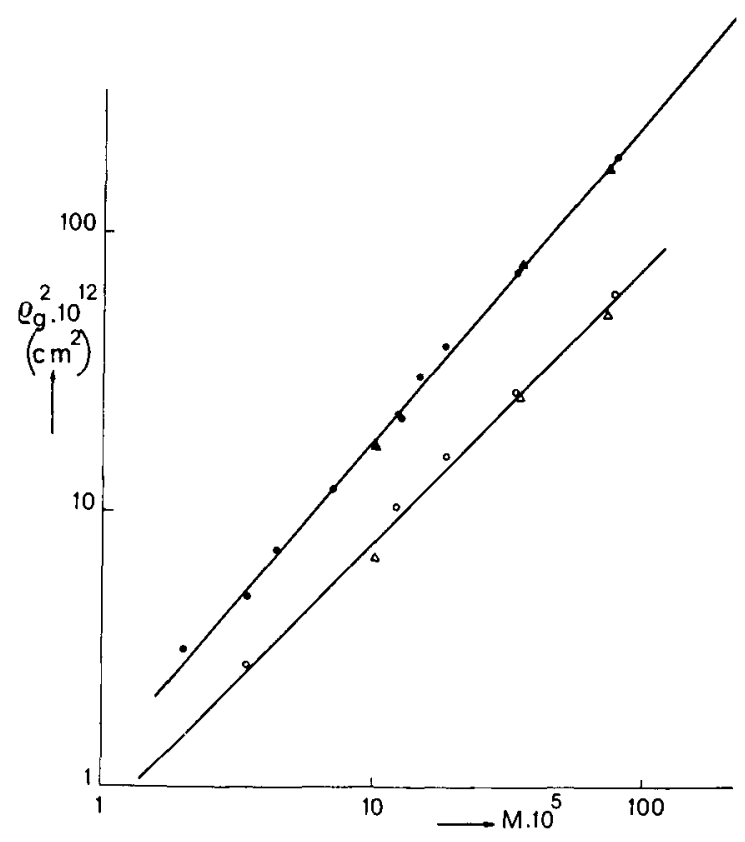

Fig. 4. Radius of gyration $\left(\rho_{g}\right)$ as a function of molecular weight $M$ : (๑) experimental values of Kato $^{18}$ in system B; (O) experimental values of Mijnlieff ${ }^{19}$ in system B; (A) experimental values of Kato $^{18}$ in system A; $(\Delta)$ experimental values of Mijnlieff ${ }^{19}$ in system A; upper solid line-eq. (32); lower solid line-eq. (32a).

approximately athermal system. Comparison between theory and experiment is facilitated by the fact that the samples of poly ( $\alpha$-methylstyrene) for which the necessary experimental information is available are nearly monodisperse.

Figure 4 gives a logarithmic plot of the values of $\rho_{g}$ vs. $M$, together with the lines representing

$$
\begin{gathered}
\text { (system A) } \rho_{g}^{2}=7.8 \times 10^{-18} M^{1.0} \\
\text { (system B) } \rho_{g}^{2}=1.78 \times 10^{-18} M^{1.17}
\end{gathered}
$$

in which $\rho_{g}$ is in cm; both $\rho_{g}$ and $M$ were determined by light scattering. ${ }^{18,19}$ The quantity $Q$ (in $\mathrm{cm}^{-2}$ ) is then, in view of eq. (15), represented by

$$
\begin{aligned}
& \text { (system A) } Q=0.192 \times 10^{18} M^{-1.0} \\
& \text { (system B) } Q=0.852 \times 10^{18} M^{-1.17}
\end{aligned}
$$

and the quantity $c(r=0)$ (in $\left.\mathrm{g}-\mathrm{cm}^{-3}\right)$, in view of eq. (12), is represented by

$$
\begin{gathered}
\text { (system A) } c(r=0)=(25.3) M^{-0.5} \\
\text { (system B) } c(r=0)=(236.9) M^{-0.755}
\end{gathered}
$$

According to eq. (14), the value of $K$ is that of the permeability $k$ at concentration $c(r=0)$ at the center of the coil. Values of $K$ can thus be read from the curves in Figure 1 when the $c(r=0)$ is known. In Tables I(a) and I(b) the values $c(r=0)$, as calculated from eqs. (34) and the corresponding $K$ values then obtained from Figure 1, have been collected for a series of molecular weights. Also given in these tables are the corresponding values of $Q$, calculated by means of eq. (33), those of $\alpha$, defined by eq. (25), those of $\Phi(\alpha)$ and $\psi(\alpha)$, read from Figures 
TABLE I

Quantities Involved in Calculating $[\eta]$ and $f$

\begin{tabular}{ccccccccc}
\hline $\begin{array}{c}M \times 10^{-6} \\
(\mathrm{~g} / \mathrm{mole})\end{array}$ & $\begin{array}{c}c(r=0) \\
\left(\mathrm{g} / \mathrm{cm}^{3}\right)\end{array}$ & $\begin{array}{c}K \times 10^{-4} \\
\left(\mathrm{~cm}^{2}\right)\end{array}$ & $\begin{array}{c}Q \times 10^{-8} \\
\left(\mathrm{~cm}^{-2}\right)\end{array}$ & $\alpha$ & $\Phi(\alpha)$ & $\Psi(\alpha)$ & $\begin{array}{c}{[\eta]} \\
\left(\mathrm{cm}^{3} / \mathrm{g}\right)\end{array}$ & $\begin{array}{c}f \times 10^{10} \\
(\mathrm{~g} / \mathrm{sec})\end{array}$ \\
\hline
\end{tabular}

\begin{tabular}{|c|c|c|c|c|c|c|c|c|}
\hline \\
\hline 0.3 & 0.0462 & & 6400 & & & & & \\
\hline 0.5 & 0.0358 & 23 & 3850 & 11.29 & 0.246 & 0.130 & 58.67 & 2274 \\
\hline 0.75 & 0.0292 & 33 & 2560 & 11.84 & 0.242 & 0.125 & 74.41 & 2812 \\
\hline 1 & 0.0253 & 44 & 1920 & 11.97 & 0.241 & 0.124 & 86.51 & 3256 \\
\hline 2 & 0.0179 & 89 & 960 & 11.70 & 0.242 & 0.127 & 120.1 & 4610 \\
\hline 3 & 0.0146 & 130 & 640 & 12.02 & 0.241 & 0.125 & 150.5 & 5709 \\
\hline 5 & 0.0113 & 215 & 385 & 12.08 & 0.240 & 0.124 & 193.7 & 7339 \\
\hline 7.5 & 0.0092 & 315 & 256 & 12.40 & 0.239 & 0.122 & 243.4 & 8825 \\
\hline 10 & 0.0080 & 415 & 192 & 12.55 & 0.238 & 0.121 & 283.3 & 10540 \\
\hline \multicolumn{9}{|c|}{ (b) System B } \\
\hline 0.3 & 0.01730 & 31 & 3329 & 9.849 & 0.252 & 0.142 & 108.7 & 1684 \\
\hline 0.5 & 0.01180 & 56 & 1831 & 9.753 & 0.252 & 0.143 & 158.3 & 2265 \\
\hline 0.75 & 0.00866 & 98 & 1139 & 8.959 & 0.257 & 0.152 & 201.5 & 2804 \\
\hline 1 & 0.00696 & 141 & 813 & 8.723 & 0.259 & 0.153 & 245.9 & 3253 \\
\hline 2 & 0.00413 & 325 & 261 & 8.523 & 0.260 & 0.155 & 407.6 & 4831 \\
\hline 3 & 0.00304 & 490 & 225 & 9.070 & 0.256 & 0.150 & 578.6 & 6360 \\
\hline 5 & 0.00207 & 900 & 123 & 9.033 & 0.257 & 0.147 & 858.7 & 8320 \\
\hline 7.5 & 0.00152 & 1480 & 77 & 8.775 & 0.258 & 0.152 & 1127.0 & 10560 \\
\hline 10 & 0.00122 & 1920 & 55 & 9.470 & 0.253 & 0.146 & 1482.0 & 12960 \\
\hline
\end{tabular}

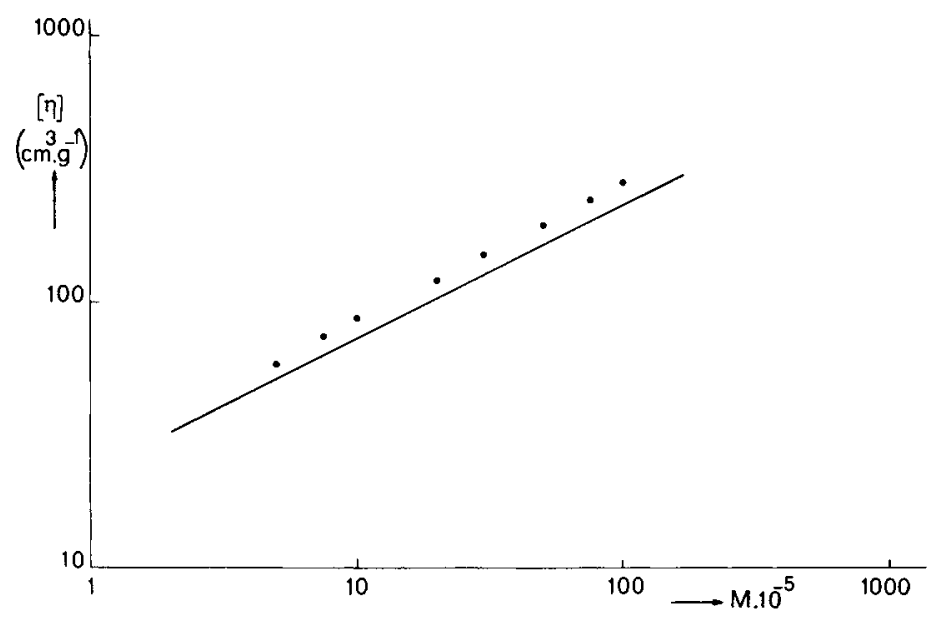

Fig. 5. Predicted and experimental values of $[\eta]$ in system A: $(\bullet)$ values predicted from eq. (27); ( - ) empirical curve (eq. (35a)) as derived by Noda $^{20}$ from experimental values.

2 and 3, respectively, and those of $[\eta]$ and $f$, calculated using eqs. (27) and (30), respectively. For $\eta_{0}$ in eq. (41), we used the following values: ${ }^{16}$

$$
\begin{aligned}
& \text { (system A) } \eta_{0}=0.00756 \mathrm{~g} \mathrm{~cm}^{-1} \mathrm{sec}^{-1} \\
& \text { (system B) } \eta_{0}=0.00553 \mathrm{~g} \mathrm{~cm}^{-1} \mathrm{sec}^{-1}
\end{aligned}
$$

The results $[\eta]$ and $f$, given in Tables $I(a)$ and $I(b)$, have been plotted in Figures 5-8. Also shown in these figures are (solid lines) the values obtained by direct measurement of these quantities. The lines in Figures 5 and 6 represent, respectively, the empirical expressions 


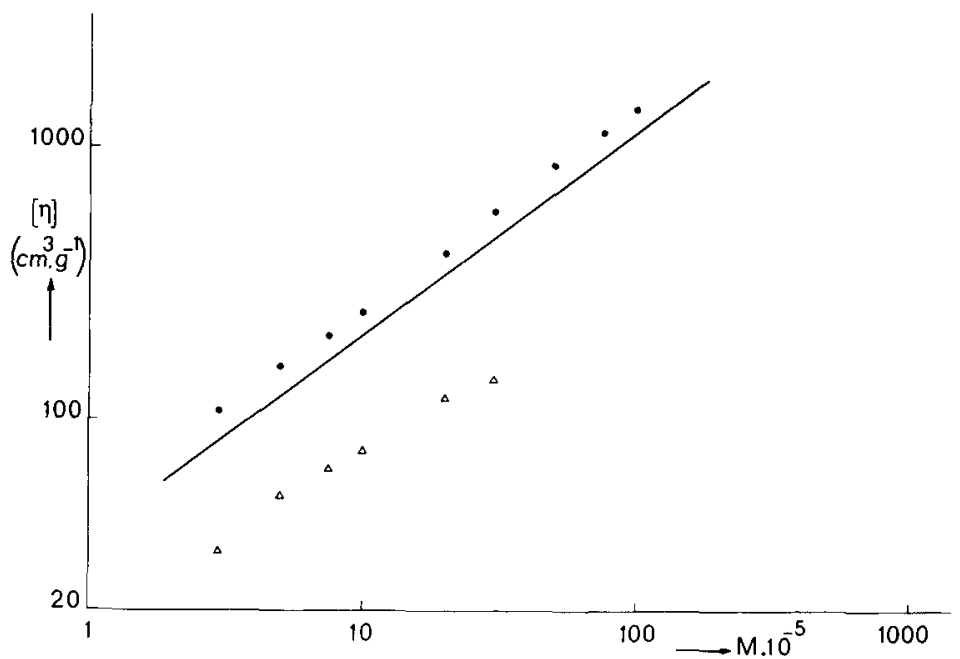

Fig. 6. Predicted and experimental values of $[\eta]$ in system B: (๑) values predicted from eq. (27); (-) empirical curve (eq. (35b)) as derived by Noda ${ }^{20}$ from experimental values; $(\Delta)$ values predicted from eq. (27), on deriving $K$ from the $h(c)$ curve pertaining to system A (see text).

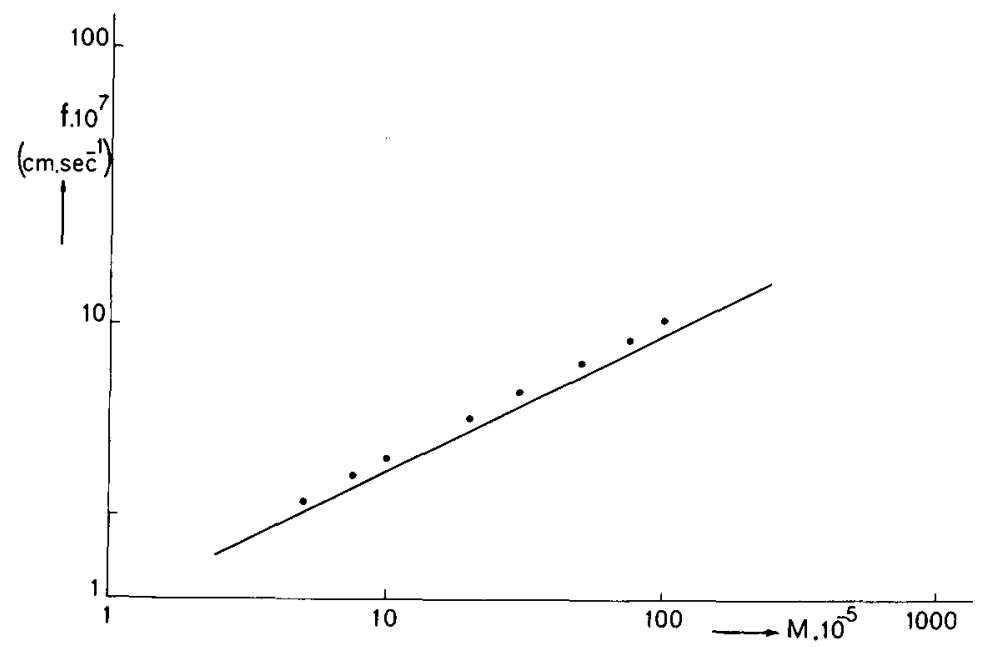

Fig. 7. Predicted and experimental values of $f$ in system A: (•) values predicted from eq. (30); (-) empirical curve (eq. (36a)) representing experimental values of Ooms. ${ }^{9}$

$$
\begin{gathered}
\left(\text { system A) }[\eta]=7.3 \times 10^{-4} M^{0.5}\right. \\
\left(\text { system B) }[\eta]=7.06 \times 10^{-4} M^{0.744}\right.
\end{gathered}
$$

derived by Noda ${ }^{20}$ from viscosity measurements.

The experimental $f$ values were calculated from experimental $s_{1}$ values by means of the equation

$$
f=\frac{M}{N_{\mathrm{Av}}} \frac{1-v_{1} / v_{0}}{s_{1}}
$$

in which $s_{1}$ is the sedimentation coefficient and $v_{1}$ and $v_{0}$ are the partial specific volume of the solute and solvent, respectively. For $\left(1-v_{1} / v_{0}\right)$ we used:16

$$
\begin{aligned}
& \left(\text { system A) }\left(1-v_{1} / v_{0}\right)=0.313\right. \\
& \left(\text { system B) }\left(1-v_{1} / v_{0}\right)=0.240\right.
\end{aligned}
$$




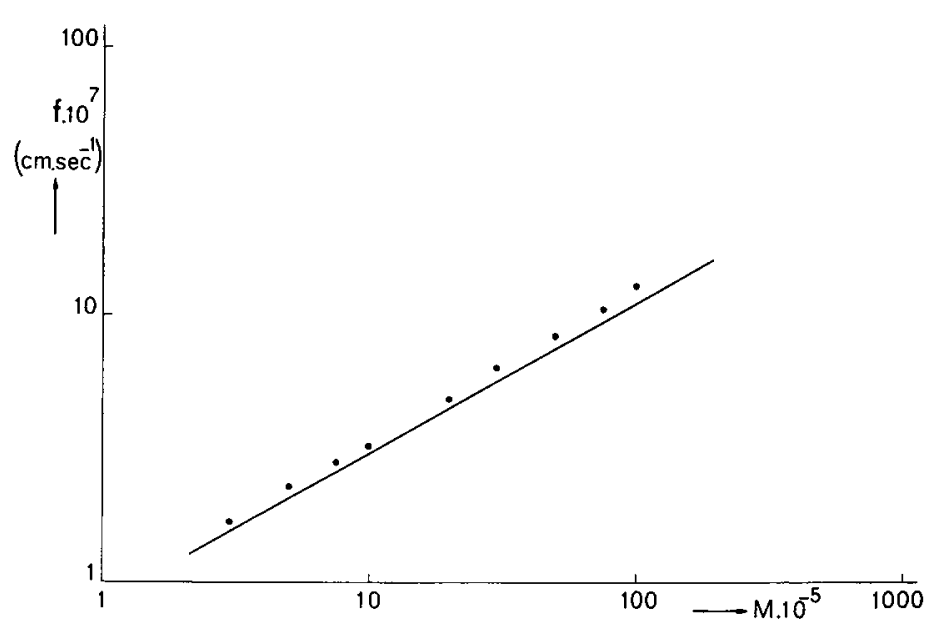

Fig. 8. Predicted and experimental values of $f$ in system B: (๑) values predicted from eq. (30); (-) empirical curve (eq. (36b)) as derived from experiments of Noda. ${ }^{21}$

It appears that $f$ can be represented by

$$
\begin{aligned}
& \text { (system A) } f=2.90 \times 10^{-10} M^{0.50} \\
& \text { (system B) } f=1.32 \times 10^{-10} M^{0.56}
\end{aligned}
$$

Expression (36a) is based on sedimentation measurements ${ }^{9}$ on three samples of poly ( $\alpha$-methylstyrene) in cyclohexane; expression (36b) follows from the empirical expression

$$
s_{1}=3.01 \times 10^{-15} M^{0.44}
$$

derived by $\mathrm{Noda}^{21}$ from sedimentation measurements on samples of poly $(\alpha-$ methylstyrene) in toluene.

The agreement between theory and experiment is satisfactory, particularly in view of the fact that there has been no "adjustment of parameters." Indeed, the values of $[\eta]$ and $f$ are predicted on the basis of knowledge of $M, Q$, and $K$. The first and second of these quantities are derived ( $Q$ is related to $\rho_{g}$ by eq. (15)) from light-scattering measurements on systems at rest; the third is derived from $k(c)$ curves obtained ${ }^{16}$ from sedimentation measurements at concentrations at which the influence of the polymer molecular weight on the sedimentation coefficient has already disappeared. None of these three quantities is, therefore, directly related to frictional properties, such as $f$ and $[\eta]$, of isolated molecules.

Whereas $M$ and $\rho_{g}$ (and thus $Q$ ) also occur as parameters in the microscopic theory of Kirkwood and Riseman, ${ }^{1}$ the quantity $K$ is a typical parameter of the porous sphere model. The value used for $K$ has a strong influence on the values predicted for $[\eta]$. To demonstrate this we have reworked the calculation of $[\eta]$ in system B using for $K$ the values derived from the $k(c)$ curve for poly $(\alpha-$ methylstyrene) in cyclohexane (system A). The values then predicted for $[\eta]$, also given in Figure 6, differ by a factor of about three from those predicted when using the proper $k(c)$ curve. The connection between the important role of $K$ and the background of the validity of the Flory-Fox equation is discussed under Conclusions.

From $[\eta]$ and $f$ and eqs. (1) and (2) the "effective hydrodynamic radii," dis- 
TABLE II

Effective Hydrodynamic Radii

\begin{tabular}{|c|c|c|c|c|c|}
\hline$M \times 10^{-6}$ & $\begin{array}{c}\rho_{g} \times 10^{8} \\
(\mathrm{~cm})\end{array}$ & $\begin{array}{c}R_{\text {eff,visc }} \times 10^{8} \\
\text { (predicted) } \\
(\mathrm{cm})\end{array}$ & $\begin{array}{c}R_{\text {eff,visc }} \times 10^{8} \\
\text { (from direct } \\
\text { measurement } \\
\text { of }[\eta]) \\
(\mathrm{cm})\end{array}$ & $\begin{array}{c}R_{\text {eff,trans }} \times 10^{8} \\
\quad \text { (predicted) } \\
(\mathrm{cm})\end{array}$ & $\begin{array}{l}R_{\text {eff,trans }} \times 10^{8} \\
\text { (from direct } \\
\text { measurement } \\
\text { of } f) \\
(\mathrm{cm})\end{array}$ \\
\hline
\end{tabular}

\begin{tabular}{crrcrr} 
& \multicolumn{5}{c}{ (a) System A } \\
0.5 & 198 & 167 & 160 & 158 & 144 \\
2 & 395 & 336 & 320 & 320 & 284 \\
10 & 883 & 765 & 715 & 731 & 638 \\
& & & (b) System B & & \\
0.5 & 288 & 232 & 213 & 217 & 197 \\
2 & 648 & 505 & 478 & 462 & 429 \\
10 & 1612 & 1328 & 1217 & 1243 & 1056 \\
\hline
\end{tabular}

TABLE III

Draining of Polymer Coils at their Center in Shear Flow and in Translation

\begin{tabular}{cccc}
\hline System considered & $\alpha$ & $f(r=0)$ & $V_{0, \text { rel }}$ \\
\hline System A, with $k(c)$ curve of system A & 12.0 & 0.39 & 0.12 \\
System B, with $k(c)$ curve of system B & 9.0 & 0.47 & 0.17 \\
System B, with $k(c)$ curve of system A & 2.2 & 0.81 & 0.54 \\
\hline
\end{tabular}

cussed in the beginning can be calculated. For three molecular weights the results, derived from the predicted, as well as from the directly measured, values of $[\eta]$ and $f$, have been collected in Table II, together with $\rho_{g}$ calculated from eqs. (32). The results confirm the experimental observation, mentioned above that the only influence of the nature of the solvent on the hydrodynamic radii stems from that on the global dimensions (for which $\rho_{g}$ is a measure) of the polymer molecule. Again, however, this simple behavior, prompting the Flory-Fox equation (eq. (5)) has a more complex background, as will be discussed under Conclusions.

The calculations also provide us with the velocity field of the solvent. We will only consider the flow at or through the center of the polymer coils; the values of $f(r=0)$, occurring in eq. (28), and of $V_{0, \text { rel }}$, defined in eq. (31), are characteristic measures for this flow. Both quantities run from unity (no influence of the polymer coil on the applied flow field, i.e., complete draining) to zero (solvent flow completely blocked, at least at the center, i.e., no draining), as $\alpha$ runs from zero to infinity.

From Tables I(a) and I(b) it can be seen that $\alpha$ depends only weakly on $M$ and is mainly determined by the type of system. For the $\alpha$ value representative of system A we take $\alpha=12$ and for system B we take $\alpha=9$. The corresponding values of $f(r=0)$ and $V_{0, \text { rel }}$ are given in Table III. Also given are the results for $\alpha=2.2$. The latter value corresponds to the case where the permeability curve pertaining to system $B$ would be the same as that for system $A$.

As was mentioned above, the observed behavior of polymer molecules appears to correspond to the nondraining limit according to the meaning which this concept has in the theory of Kirkwood and Riseman. From Table III it will be 
clear that this does not at all imply that the polymer molecules, not even at their center, are essentially nondraining. We come back to this point in the next section.

\section{CONCLUSIONS AND DISCUSSION}

\section{General Conclusions}

In this paper the intrinsic viscosity $[\eta]$ and the translational friction coefficient $f$ of the polymer molecules in solution have been calculated on the basis of the porous sphere model. Calculated and directly measured values are compared in Figures 5-8. They agree within 10 to $20 \%$ This is satisfactory in view of the fact that no a priori information on $[\eta]$ or $f$ has been used to arrive at the result. The three parameters by which $[\eta]$ and $f$ are determined are $M, Q$ (or $\rho_{g}$ ), and $K$. The essential role of $K$ has already been emphasized. The fact that the permeability curves - from which $K$ could be read-were available for both systems considered is, we think, the major reason why theory and experiment agree quite well. As $k(c)$ curves are readily obtained ${ }^{16}$ from sedimentation measurements at finite concentrations, the theory can easily be applied to systems other than those discussed here.

\section{Possible Refinements of the Theory}

The results, as we mentioned above, are quite sensitive to the value adopted for $K$. Above, when we discussed the model, the function $k(r)$, which had to be known to permit the solution of eq. (8), was approximated by eq. (13), in which $K$ denotes the permeability at the center of the coil. This procedure is justified if the right-hand side of eq. (13) represents exactly the shape of the $k(r)$ curve obtained by combining the $k(c)$ curve as measured with the $c(r)$ curve that corresponds to the function (in our case eq. (12)) adopted for $c(r)$. However, if we let $c(r)$ be given by eq. (12), $K \exp \left(Q r^{2}\right)$ does not exactly coincide with the $k(r)$ curve obtained in the way described above. Making the choice

$$
K=k(r=0)
$$

means that we make the two $k(r)$ curves coincide at $r=0$. Other choices, which for physical reasons might be more attractive, can be imagined.

The validity of eq. (12) should be investigated more closely. Although there is no simple relation between the distribution function $c(r)$ and the distribution function of end-to-end distances, it may be anticipated that the non-Gaussian character of the distribution function for end-to-end distances in system $B$ (a system far from theta conditions) will also play a part in the distribution function $c(r)$. Only when this second point has been clarified would it make sense to use a more refined expression; say, a three-parameter expression for $k(r)$. In this context we note that in the earlier calculation ${ }^{9}$ of $f$ using the porous sphere model, the necessity of adopting an analytical expression for $k(r)$ was avoided by considering the polymer coil as consisting of (thin) shells, each of which was assigned its own permeability. This method, however, is elaborate and becomes very difficult when intrinsic viscosities have to be calculated. 


\section{Connection between the Molecular and the Porous Sphere Model}

The role of the $\rho_{p} \zeta$ in the molecular model (eq. (11)) is the same as that of $\eta_{0} / k$ in the porous sphere model (eq. (8)). However, the available information on $\eta_{0} / k(r)$ does not yet tell us how the product $\rho_{g} \xi$ has to be divided. Even stronger, as soon as $k(r)$ is no longer inversely proportional to $c$-and this is what is found at higher concentrations - "the effective friction coefficient $\zeta$ " becomes a quantity that depends on $\rho_{p}$. A quantitative prediction of this dependence from a molecular model will be difficult.

Because eq. (8), the hydrodynamic equation we solved, has the same form as eq. (11), derived from the molecular theory, we think that our results on the solvent flow correspond to about the best that might be independently derived from the molecular theory. And for the time being, the results contain more detail than has heretofore been produced by a molecular theory. We note, in particular, the marked influence of solvent on permeability. This influence as yet finds no counterpart in predictions by any molecular theory on values of $\rho_{p}$ and/or $\zeta$ in different solvents.

\section{Flory-Fox Equation}

The Flory-Fox equation is beautifully simple because it is valid in poor as well as in good solvents; but the background of this simplicity is not at all simple. In order to see this, let us follow the changes in a polymer molecule when it is transferred from a poor to a good solvent. The best known effect of such a change of environment is an increase of the radius of gyration. This increase leads to a change in $c(r)$, which can be described approximately with the aid of eq. (12). The corresponding change in $K$ is found by applying eq. (14). If we take poly ( $\alpha$-methylstyrene), $M=2 \times 10^{6}$, as an example, its $\rho_{g}$ on transfer from cyclohexane to toluene increases by a factor of about 1.4. If we then use eq. (14) in order to find the corresponding new value of $K$, while assuming that the $k(c)$ curve is still the same as that in cyclohexane, we arrive at a value for $K$ equal to

$$
K=130 \times 10^{-14} \mathrm{~cm}^{2}
$$

which ultimately leads to a value for $[\eta]$ (see Fig. 6) of

$$
[\eta]=121.4 \mathrm{~cm}^{3} \mathrm{~g}^{-1}
$$

It is clear that this result would not at all lead to a solvent-independent value of the coefficient $\Phi$ in eq. (5). However, if we derive the values for $K$, as we should, from the $k(c)$ curve in toluene, we find

$$
K=325 \times 10^{-14} \mathrm{~cm}^{2}
$$

which leads to (see Table I(b)):

$$
[\eta]=407.6 \mathrm{~cm}^{3} \mathrm{~g}^{-1}
$$

This is not far from the experimental value

$$
[\eta]=344.2 \mathrm{~cm}^{3} \mathrm{~g}^{-1}
$$

and leaves the value of $\Phi$ in eq. (5) essentially the same. The validity of the Flory-Fox equation stems, therefore, from the fact that two large effects, one 
of which is known (influence of solvent on $\rho_{g}$ ) and one of which is not very well known (influence of solvent on permeability), affect $[\eta]$ in opposite directions and, by chance, approximately compensate. The same statement can be made about the degree of draining. If in the example considered above, the $k(c)$ curve for toluene had been supposed to be the same as that for cyclohexane, the degree of draining in shear flow (the quantity $f(r=0)$ ) would have been increased (see Table III) from about 38 to about $81 \%$. Only because the $k(c)$ curve lies much lower in toluene than in cyclohexane does the degree of draining increase to no more than $47 \%$ (see Table III).

\section{Solvent Influence on $k(c)$ Curves}

The possibility of predicting $[\eta]$ and $f$ rather accurately was shown to be closely related to the fact that $k(c)$ curves were available from independent measurements. An important feature of these curves was the strong dependence of their level (see Fig. 1) on the solvent quality. Some years ago this influence was explained ${ }^{16}$ in terms of a tendency for local association of polymer chains depending on the type of solvent, and the observed ${ }^{16}$ dependence of the $k(c)$ curves on temperature was seen to support this statement. Recently this tendency for association has also been derived from purely theoretical considerations. ${ }^{22}$

In this context we briefly discuss the influence of temperature. In the approximately athermal solutions in toluene, the $k(c)$ curve, up to about $100^{\circ} \mathrm{C}$, is hardly affected by temperature. ${ }^{16}$ Because the $\rho_{g}$ values will not change very much either, no appreciable effect of temperature on $[\eta]$ can be expected. In solutions in the poor solvent cyclohexane, on the other hand, the whole level of the $k(c)$ curve decreases sharply and approaches that in toluene if we move away from the theta temperature. ${ }^{16}$ In addition, $\rho_{g}$ increases with increasing temperature. The influence of temperature on $[\eta]$ stems from a combination of the two above-mentioned effects. In predicting $[\eta]$ values this necessitates only that $k(c)$ curves and $\rho_{g}$ values pertaining to the proper temperature must be used.

\section{References}

1. J. G. Kirkwood and J. Riseman, J. Chem. Phys., 16, 565 (1948).

2. H. Yamakawa, Modern Theory of Polymer Solutions, Harper \& Row, New York, 1971, (a) Chap. 6; (b) Chap. 7, esp. p. 380; (c) Chap. 2, esp. p. 26.

3. H. Morawetz, Macromolecules in Solution, Interscience, New York, 1965, (a) p. 304; (b) p. 274.

4. M. Kurata and W. H. Stockmayer, Fortschr. Hochpolym. Forschung, 3, (1963), (a) p. 198; (b) p. 225; (c) p. 226 .

5. P. Debye, Phys. Rev., 71, 486 (1947).

6. P. Debye and A. M. Bueche, J. Chem. Phys., 16, 573 (1948).

7. H. C. Brinkman, Proc. Acad. Sci., Amsterdam, 50, 618 (1947).

8. H. C. Brinkman, Physica, 13, 447 (1947).

9. G. Ooms, P. F. Mijnlieff, and H. L. Beckers, J. Chem. Phys., 53, 4123 (1970).

10. F. W. Wiegel and P. F. Mijnlieff, Physica, in press.

11. F. W. Wiegel and P. F. Mijnlieff, Physica, 85A, 207 (1976).

12. B. U. Felderhof and J. M. Deutch, J. Chem. Phys., 62, 2391 (1975).

13. B. U. Felderhof and J. M. Deutch, J. Chem. Phys., 62, 2398 (1975).

14. B. U. Felderhof, Physica, 80A, 63 (1975).

15. B. U. Felderhof, Physica, 80A, 172 (1975).

16. P. F. Mijnlieff and W. J. M. Jaspers, Trans, Faraday Soc., 67, 1837 (1971).

17. J. M. Burgers, in Second Report on Viscosity and Plasticity (Proc. Acad. Sci., Amsterdam (II)), vol. 16, 1938, p. 128 . 
18. T. Kato, K. Miyaso, I. Noda, 'T. Fujimoto, and M. Nagasawa, Macromolecules, 3, 777 (1970).

19. P. F. Mijnlieff, D. Coumou, and J. Meisner, J. Chem. Phys., 53, 1975 (1970).

20. I. Noda, K. Mizutani, T. Kato, T. Fujimoto, and H. Nagasawa, Macromolecules, 3, 787 (1970).

21. I. Noda, S. Saito, T. Fujimoto, and M. Nagasawa, J. Phys. Chem., 71, 4048 (1967).

22. P. G. de Gennes (Collège de France, Paris), private communication.

Received November 18, 1976

Revised July 20, 1977 